\title{
Network Support for Integrated Design
}

\author{
Daizhong $\mathrm{Su}^{1,3}$ and Xiaoan $\mathrm{Chen}^{2}$ \\ 1 Professor of Design Engineering, School of Engineering, The Nottingham Trent University, UK; \\ Cheung Kong Professor, State Key Laboratory of Mechanical Transmission, Chongqing University, China. \\ 2 Professor of Manufacturing Engineering, State Key Laboratory of Mechanical Transmission, Chongqing \\ University, China. \\ 3 Corresponding author, School of Engineering, The Nottingham Trent University, Burton Street, Nottingham, \\ NG1 4BU, UK; E-mail: Daizhong.su@ntu.ac.uk; http://domme.ntu.ac.uk/mechdes
}

\begin{abstract}
A framework of network support for utilization of integrated design over the Internet has been developed. The techniques presented also applicable for Intranet/Extranet. The integrated design system was initially developed for local application in a single site. With the network support, geographically dispersed designers can collaborate a design task through out the total design process, quickly respond to clients' requests and enhance the design argilty. In this paper, after a brief introduction of the integrated design system, the network support framework is presented, followed by description of two key techniques involved: Java Saverlet approach for remotely executing a large program and online CAD collaboration.
\end{abstract}

Keywords: Internet, Network supported system, collaborative design, Design integration, CAD

\section{Introduction}

With the development of computer technologies, the networks (Internet, Intranet and extranet) have been widely applied in business, engineering, education and other areas. In the IT-related fields such as e-business, e-commerce, e-factory, e-manufacturing and virtual institution, the network technique plays an important role.

This fast growing technology has been attracting great attention from researchers, industrialists, businessmen and people with various background. 'Sooner or later every organization will use the Internet for some facet, large or small, of its business' [1]. In the prestigious collection [2], seven applications/case studies of Internet-based engineering are presented covering the subject areas of resource discovery, Internet as a tool for engineering design, engineering education and training, Web-centered manufacturing and comprehensive technique for network security. Research and literature publications in this area are continuously and increasingly emerging, for example, Gausemeier in 1996 reported an European Union supported international collaborative research project of global engineering network [10], Huang et al made contributions to design for $\mathrm{X}$ over the Internet in 1997 [3], Shackleford and Proctor developed Java-based tools for real-time control systems in 1998 [14], Su and Goliski implemented a mechanical design system on the Internet in 1999 [13], Peng and Hall in 2000 conducted the research of integrated design and manufacture for SMEs through a remote web-centered interface [15], Mauer in 2001 presented the new aspects of e-learning [16], and more and more could be mentioned.

An integrated design system has been developed [11][12], which integrates various stages within the total design process [4] including product design specification, conceptual design, detail design and manufacture. The system greatly speeds up the design process and reduces time-to-market. It was initially developed for a design task to be conducted at single site. With nowadays rapid development of the network techniques, a prototype framework is developed by the authors to utilize the integrated design system on the Internet. With the network support, geographically dispersed designers can collaborate a design task through out the total design process, quickly respond to clients' requests and enhance the design argilty.

Although the work implemented is for the Internet, the techniques developed for the network support for integrated design are generally applicable for the Intranet and Extranet.

In the following sections, after a brief introduction of the integrated design system, the network support framework is presented, followed by description of two key techniques involved: Java Saverlet approach for remotely executing a large program and online CAD collaboration.

\section{A Prototype Framework for Network Supported Design Integration}


In this section, the integrated design system is briefly described first, and then a prototype network support framework is presented with information how the integrated design system can be utilized over the Internet. The key techniques involved are also highlighted.

\subsection{The integrated design system}

In the system, there are two types of integration: the integration of the total design process and the integration of various software techniques/packages (including programs) into a single environment to conduct the design. Figure 1 shows the integrated design process and relevant software techniques/packages involved at each design stage.

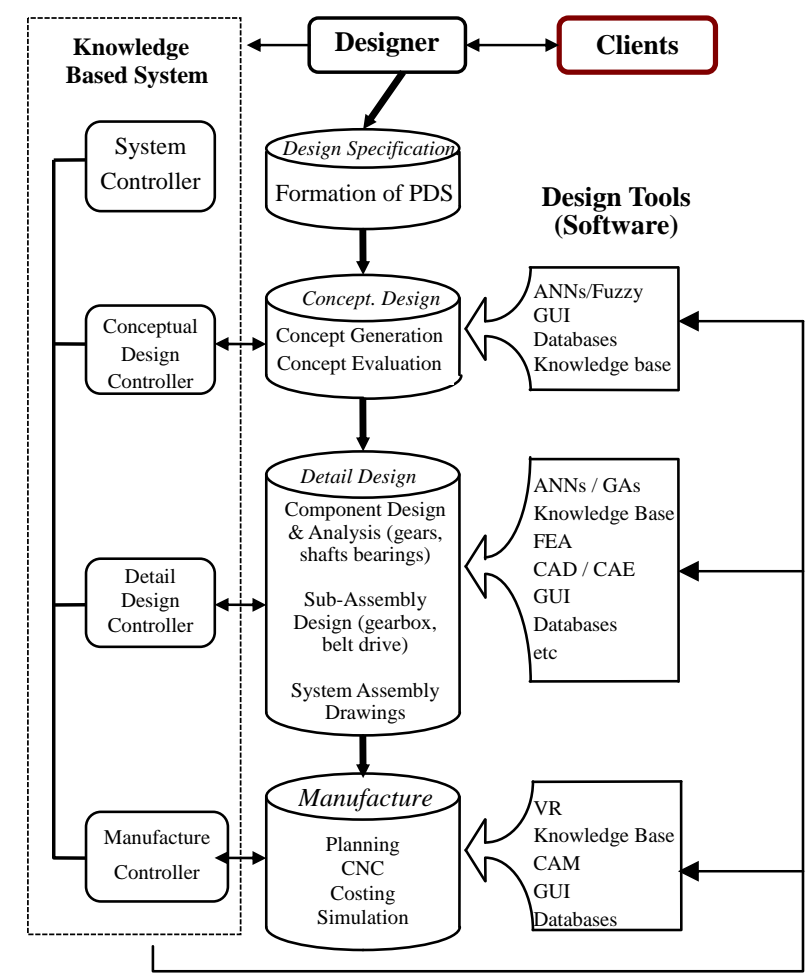

Figure 1 The intelligent hybrid system for integrated design

The designer interacts with the knowledge-based system (KBS). The design and manufacture expertise is captured by the KBS and artificial neural networks (ANNs), while the other tasks within the design process, such as numerical analysis, engineering drawing, data processing, optimisation and simulation are conducted using relevant CAD/CAE/CAM packages, and genetic algorithms (GA) and virtual reality (VR) software. The graphical user interfaces (GUI) are developed using advanced hypermedia techniques. The KBS communicates with the others and works as a coordinator controlling the whole process. Within the system, the following activities are integrated throughout the process of total design:

- $\quad$ conceptual design: formulation of product design specification, concept generation and evaluation;

- $\quad$ detail design: analysis, sub-assembly/component design and assembly, engineering drawings, design retrieval;

- $\quad$ manufacture: process planning, costing and CNC manufacture.

As an example to illustrate the approach, an integrated system for total design of mechanical power transmission systems has been developed to perform the following tasks [3]:

a. Formulation of Product Design Specification (PDS). 12 PDS items, such as orientation of input/output shafts, manufacture cost, etc., are considered. It is possible to extend the system to aid more PDS items.

b. Conceptual Design. The concepts to be constructed by the prototype IHS fall into the following range:

- stages of the transmission: one, two or three;

- $\quad$ orientation of input/output shafts: parallel, cross and perpendicular;

- components at each stage of the transmission: seven types of components including gears, belts and trains.

c. Detail Design. The system can conduct the detail design of gearboxes, belt drives or combination of both, including gear strength analysis, bearing selection, shaft design, case design, belt and pulley selection, design 
optimisation, component and assembly drawings, and parametric design of components.

d. Manufacture. The system can perform the following tasks: cost analysis, process planning for the manufacture of major components, and CNC programming for manufacture of shafts

For further information of the integrated design system, see [11,12,13]. To conduct a large scale design task, it is required for team members to collaborate with each other. However, sometimes they are not all at one location. Therefore a framework has been developed for geographically dispersed team members to conduct an integrated design task over the Internet/Intranet using the integrated system, which is described in the next section.

\subsection{Framework of the network-supported system for integrated design}

The framework of the network-support for utilization of the integrated design system mentioned above is shown in Figure 2, and is detailed below.

The clients from anywhere in the world can submit their requirements for a particular design task to the main design site via the Internet. The marketing and commercial representatives at different locations also send customers' feed back about the design to the main design site in the same way. This enables the design team get the information effectively and quickly to formulate the product design specifications.

The design is conducted by a geographically dispersed team whose members collaborate over the Internet/Intranet. The chief designer is at the main design site, where the KBS and the main body of the integrated design system are located; the chief designer interacts with the KBS and communicates with other designers via the network to control the design process. Each of the other designers has special contributions to the design at certain design stage. The designers can also conduct online discussion and online-modification of the design. A technique for this has been developed by this research which is to be mentioned latter.

The design tools, i.e., software packages/programs, such as CAD, CAE, finite element analysis (FEA), virtual reality (VR) and artificial intelligence (AI), are located at different sites -- some of the tools may be with the designers, while the others may be at individual web sites; all of them are accessible for the designers via the Internet/Intranet. This enables the design team to share the resources amongst the team members.

At the conceptual design and detail design stages, it is necessary to choose certain items, such as standard components and materials, from the supply chain. Within the framework, the search for suitable suppliers is conducted via the Internet. To speed up the search, not only a powerful Internet search engine is needed, but also it is important for the suppliers to present their product information effectively. An important issue regarding the latter aspect is how the providers provide informative online electronic catalogues in a user-friendly manner to designers.

The Internet link between the designer team and the manufacturer adds a new feature to the integrated design system, which is beneficial for both sides. For example, not only they communicate with data transfer, but also the manufacturer can view the drawings and modify the drawings online, and can run the virtual manufacture software, which is located at the other site, to simulate the manufacture of the designed product before actually making it.

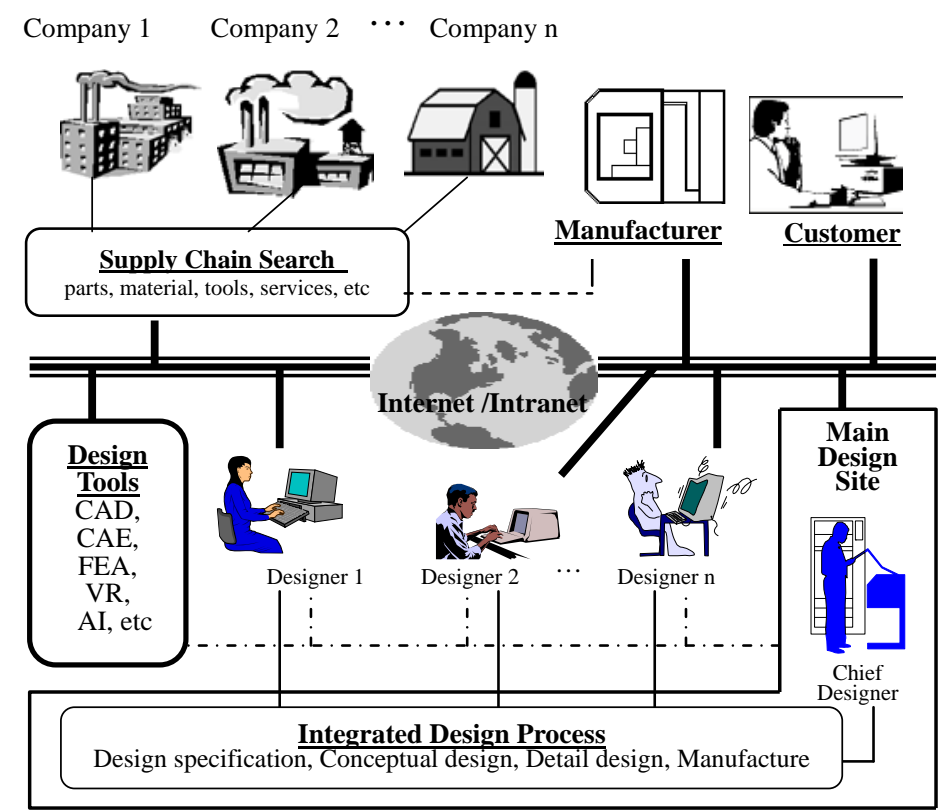

Figure 2 Network supported integrated design 


\section{Internet Related Techniques}

Obviously, to implement the network supported system, considerable amount of work is involved. At current stage of this research, the following Internet related techniques are considered:

\subsection{Formulation of the product design specifications via the Internet}

At the main design site, the product design specifications (PDS) are formulated based on the client's requirements. It is often that the client's requirements submitted may not contain all the information required to complete the PDS. The following are considered to overcome this problem:

- To establish an interactive way via the Internet for the designer and the client to communicate with each other. This can be achieved using Java script, Java Applet and HTML for the client to submit their requirements in a pre-designed form, and at the sever side, a simple knowledge-based system checks the form (in some special cases, the human designer maybe involved to check); if the form is incomplete, then return to the client for re-submission.

- A fuzzy program to deal with the incomplete information to generate the PDS. An example of such a system can be seen in [5].

- $\quad$ In some cases, the combination of both the above may be necessary.

\subsection{Integration of online electronic catalogues.}

As mentioned before, to provide user-friendly and informative online electronic catalogues is an important matter for the network-supported system. As an example, an online gear catalogue has been developed in this research.

Currently there are a number of electronic catalogues available on the Web, however, many of these catalogues are static. They are made up of a number of simple HTML pages with hard coded tables of data and images, which are not flexible for change. Some catalogues have the option of downloading the drawing in Data Exchange Format (DXF) for a few components, but the DXF files are hard coded which not only are not flexible to adopt changes but also would take up a lot of space on the server if it were required to provide DXF for all the components, especially when the data is large.

This research integrates an electronic catalogue for a gear stock company into the Internet. In addition to retrieving the appropriate table of data, the user would have the option of downloading the drawing of the selected gear in DXF format which could easily be imported into any CAD software that can read DXF files such as AutoCAD. Deferent from the existing electronic catalogues, the drawings are created dynamically on the server, which reduces the required spaces dramatically and easy to adopt changes. A structure based on a combination of Java Applet, Java Database Connectivity (JDBC) and Java Servlet has been utilised to accomplish the task.

The online catalogue is particularly important for the gear manufacturers for selling their product. It is also beneficial for the engineers working with CAD packages. The software would save hours of time creating the individual drawings. There would only exist one copy of the database, which is located on the server. Any changes, such as addition or removal of a certain data or gear dimension, could be made at any time. This means that the user would always have access to the up-to-date version of the database. Customers could easily connect to the web site, retrieve the data and create the DXF file. For further detail, see [6]

\subsection{Remotely execute a large size program over the network}

The program located in the owner's web site and the collaborative designers can remotely run the program from other locations without downloading it. This enables the best utilization of the resources amongst the geographically dispersed team members and secures both the copyright and updating. To illustrate the approach, a large size program of gear design optimization has been utilized. The program implements genetic algorithm to optimize up to 9 design parameters with 5 objective functions for spur and helical gear design. It consists three parts: a friendly graphical user interface, a genetic algorithm program, and a numerical analysis program for gear strength calculation to the British Standard 436 [7]. The optimization program is located and executed on the server-side and the results are sent to the client-side after completion of the design optimization. It works in a multi-users environment, i.e., several users can remotely run the program at the same time. Two methods 
for this purpose have been developed: common gateway interface (CGI) [17] and Java Servlet [18]

CGI is a program located on the server and can communicate with the HTML forms. It can be written in any programming language such as Java, $\mathrm{C} / \mathrm{C}++$ and perl which can read from standard input, write to standard output, and read environment variables. The data that the CGI program receives from the form is in the form of one long string that needs to be parsed first. Subsequently the CGI program processes the data and sends the results back to the user, in the form of an HTML page.

The CGI programs that have been developed for this research have been written in C++ language. There are two different methods of sending the data to the server: GET and POST. The GET method sends its data as command line input, i.e. as one long string assigned to an environment variable, whereas the POST method sends all data values as one line of text to standard input device which must be read within the program [19]. The amount of data that can be retrieved from a GET is considerably smaller than that from a POST. Since the number of data needs to be obtained from the user is considerably large, it was decided to use POST to transmit the data.

Because CGI support was built into every web server on the market, CGI was a popular choice for development tools and applications that could add dynamic capabilities to a Web site. But CGI has drawbacks, which has been experienced during this project. A new process needs to be created for each request. This leads to performance problems at popular Web sites that handle requests from multiple users. In addition, this package requires great time for execution.

Java Servlets are small, platform-independent server-side programs that also extend the dynamical function of the web server. Servlets run on a Java-enabled web server and can provide the web service in the module of request-response. Generally they could implement the same functions as CGI, but they have their own properties.

The main property of Java Servlets is to improve the speed issues of CGI. Servlets solves the performance problem by executing all requests as threads in one process. It starts a new thread (rather than a new process) with each client request.

The so-called processes are different programs, such as WORD and Excel, running in the same computer system, which have different addresses and spaces. Context switch between different processes and changing currently running process are complicated. The communication between different processes is expensive and limited. Even though many processes can be running simultaneously, only one process can be communicated with.

Thread is a control stream with one order in a process, also called lightweight process. Threads share the same address and space and construct one big process. The communication between threads is simple and effective, and context switch is fast and is part of the whole program. Threads could be executed separately. Running several threads simultaneously in one process could be used to finish different tasks. Multiple threads provide the interactive power of the program, more GUI and more powerful server functions.

The essential reason why the Java Servlets could improve the performance defaults of CGI is that Servlets are multithreadeds and run within the process of the Servlet server. A process context switch is not required to handle each Servlet request. When the first time that a Servlet is requested, it is loaded into the Web server's memory space. Subsequent client requests for the Servlet result in calls to the Servlet instance in memory.

Servlets offer many other added benefits to the developer, including ease of development, fast throughput and response, inter-server communications, and all of the features inherent in Java.

\subsection{Instantaneously online co-modifying CAD drawings}

Within the framework of the network supported system, the design team members from different locations can discuss/exchange information instantly over the network, and the changes made on the CAD drawings by anyone in the design team can be displayed simultaneously on the screen of each member's computer and the designers communicate not only by typing the message via the keyboard but also by voice and video using the Net-Meeting software. Further detail about this is given the following section.

\section{Online CAD Collaboration}

Nowadays, CAD drawings are essential means for designers to express their designs. In order to conduct the integrated design over the Internet/Intranet, it is necessary to provide an online framework to enable geographically dispersed team members to discuss and to modify CAD drawings simultaneously.

Currently, the existing CAD packages, for example AutoCAD and Pro Engineer, are restricted to a single location application only. Taking AutoCAD as an example, this research has developed a method to extend the 
traditional single location CAD systems for multi-location application over the Internet/Intranet, providing a framework for designers at different locations to online modify CAD drawings and discuss related issues.

\subsection{System structure and software environment}

The system structure is shown in Figure 9. In the system, the software NetMeeting [9] plays a key role of connecting an individual site to the Internet, with multimedia functions of audio/video, message board and "chat"; the Dynamic-Link Model (DLM) carries out the tasks of dynamic data processing and links with the AutoCAD and NetMeeting. The designer operates the AutoCAD to produce drawings and makes changes which are instantaneously sent, with the aids of DLM and NetMeeting, to the collaborative designers at different sites. With NetMeeting's multimedia functions, the designers can communicate online with each other in the ways of speaking, writing message on the board, and seeing each other on the screen during the collaborative design process.

Site 1

Site $2 \quad \ldots \ldots \quad$ Site $\mathbf{n}$

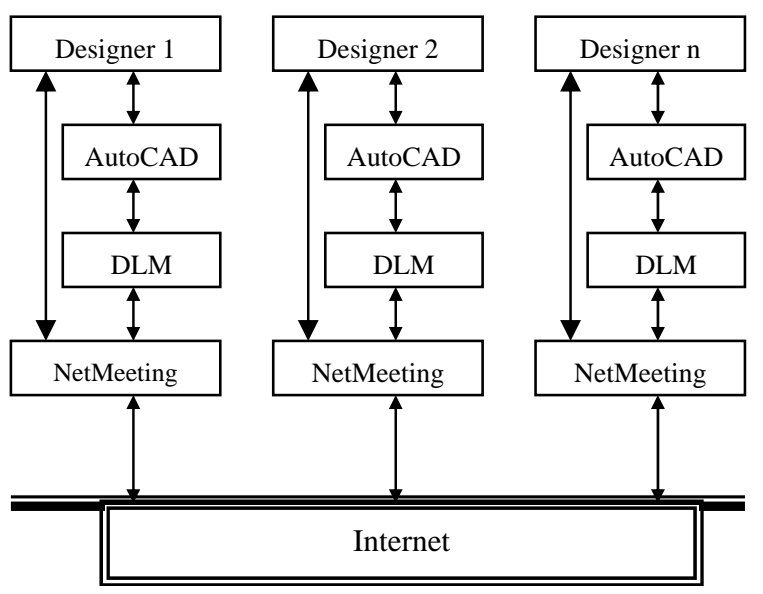

Figure 9 Structure of the online collaborative CAD system

The DLM achieves its communication with the AutoCAD via ObjectARX. The ObjectARX is a powerful development tool of AutoCAD, which is released with AutoCAD by AutoDesk Co. The ARX program adopts the object-oriented mechanism of application program in C++. The ARX application shares AutoCAD's ID space in the same way of the DLM so that the programmer of AutoCAD User's Development can directly define its internal data structure, graphic programs and user commands by using the open architecture of AutoCAD.

NetMeeting provides an open, extensible platform for real-time communications, offering audio, video, and data conferencing functionality. Developers can leverage NetMeeting capabilities to integrate real-time multipoint data conferencing communication into existing conferencing solutions. Developers can:

- $\quad$ Create custom conferencing solutions to enhance service offerings.

- Integrate conferencing functionality into existing Web pages.

- $\quad$ Extend the features of NetMeeting.

NetMeeting interacts with the DLM via the Component Object Model. The use of the component objects of NetMeeting needs the development tool which is part of the NetMeeting's Software Developer Kid (SDK). The SDK is an executable file 'NM3SDK.exe', which can be downloaded from the Microsoft web site [9]. The internal function models of NetMeeting are divided into some component objects, each object accesses one interface, and each interface supports some functions. Application program can access these functions by the interface pointer.

\subsection{Dynamic link model (DLM)}

NetMeeting has the network functions as mentioned above, but does not have CAD drawing capabilities; while AutoCAD is a powerful CAD package, but does not have network functions required for this project. The DLM's links the two packages to make it available to instantaneously transmit the CAD drawings over the Internet. 
During the process of online modification of CAD drawings, the initial drawing, was distributed to each networked AutoCAD first, then in the subsequent online modification of the drawing, only the changed part of the drawing, i.e., the modified, added or deleted entities, is transmitted over the Internet. This greatly enhances the speed of updating the same drawing on each computer in the network.

The DLM is written in Visual C++. Figure 10 shows the major function of the DLM. To achieve the function, the DLM works in four steps as detailed below:

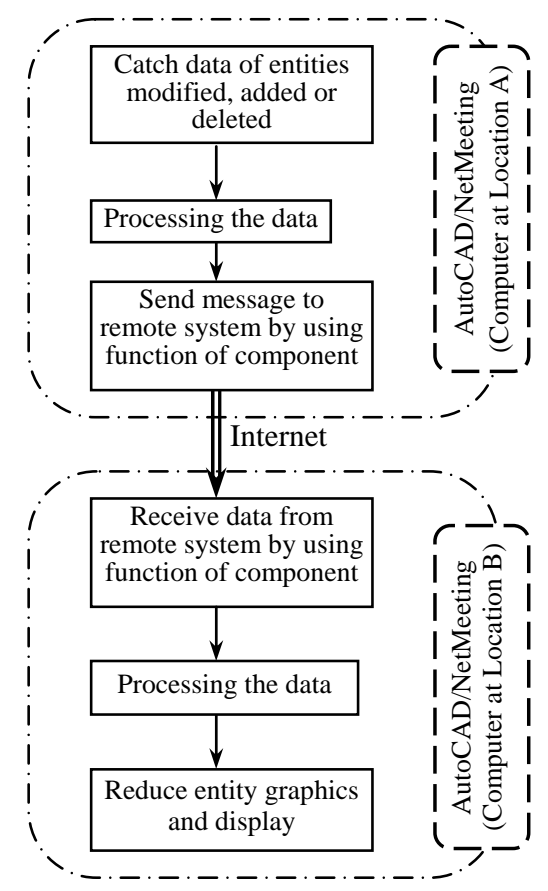

Figure 10. Major function of the DLM

The first step is the monitoring and catching the entity changes of the drawings in AutoCAD, including modifying, adding or deleting an entity.

AutoCAD Database $(\mathrm{AcDb})$ is a set of graphics data arranged with certain structure. Data stored at AcDb is called as $\mathrm{AcDb}$ object, which is a basic part forming AutoCAD drawings including visible geometrical entity and invisible non-geometrical objects etc. While an AutoCAD drawing is opened, a database is produced automatically in the system, which includes all data of the drawing. While using a command of AutoCAD to draw an entity, the record related is written into the database. A database special reactor is developed to monitor the events occurred in the database, for instance, an object is added to the database or is modified, deleted etc., and the information related to the object is obtained by the object's pointer.

In the $\mathrm{AcDb}$ the information of the entity obtained is stored in the result buffer. The pointer of the result buffer of a selected entity can be obtained by function "struct resbuf*ads-entget (ads_name ent)". The result buffer in the program is written as follows:

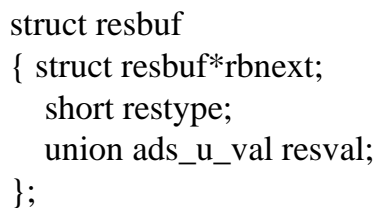

The statement, "struct resbuf” (result buffer) includes 3 segments:

- $\quad$ rbnext--pointer variable of directing next result buffer.

- $\quad$ restype--type indicate variable about the equivalent digit segment "resval" value on the node of the result buffer.

- resval--node value variable stored in the result buffer. "union ads_u_val” is a synthetic type variable.

Because there is pointer variable in the result buffer "rbnext" which directs to the next result buffer, a whole set of result buffers can be chained as a chain list. Each buffer node describes the information of one 
item of an entity, such as, colour, line type, etc. A whole set of result buffers is chained to describe the complete information of whole entity.

The second step is to handle the information of entity change.

The information handle is to extract the information of the entity from result buffers and then to transform it into the form of a byte array, which corresponds with the function finished in DXF file output. Based on the value of "restype", type variable of the equivalent digit segment "resval" value of the node in the result buffer can be determined, the "resval" value can be transformed into character mode and the front and rear are added marks, then are written in the byte array.

The third step is to send and to receive the information.

To deliver and to receive information are accomplished by using the NetMeeting's element function, including set up multi-media conference and network link among AutoCAD packages. The element function is indicated in the DLL.

The information transferred via the Internet is received by NetMeeting, which is a byte array mode. The byte array can not be treated as entity and hence must be processed. The processing procedure is similar to the way of obtaining information from DXF files. The information obtained is used to create a linked-list of the result buffers. The function "struct resbuf*ads_newrb(int V)" is used to create the single node of the result buffers, where "V" is the group code of DXF.

The last step is to reduce the entity graphics in accordance with the result buffers, The function "int ads_entmake(struct resbuf *ent)" creates a new entity instructed by the parameter "ent" in the result buffer and adds into the drawing file database. Then the graphic entities are simultaneously transmitted among the geographically distributed AutoCAD design system.

\subsection{A case study}

A case study for the system mentioned above has been conducted. It includes 3 computers with PIII400 processors located at three sites and linked to the Internet. Each of them are installed with AutoCAD version 14, NetMeeting version 3.0, the DLM developed by the authors and the network environment TCP/IP. Each computer also is equiped with video and audio facilities such as video a camera and a microphone.

The system is operated in the following procedure:

- $\quad$ Each designer starts AutoCAD in his/her local computer

- $\quad$ Load the DLM in AutoCAD, then NetMeeting runs automatically

- With NetMeeting, one of designers, acting as the leader, calls other designers to attend the collaborative design (setup Internet conference link)

- The leader opens a drawing or draws a new one in AutoCAD in his/her computer; at this moment, the drawing is sent automatically to the computers of the participants and is automatically displayed in AutoCAD in their local computers.

- Then, the participants can discuss this drawing using functions of audio/video, whiteboard and "chat" in the system

- Meanwhile, the participants can modify (add or delete entity) this drawing in their local computer. Only the entity modified in local AutoCAD will be transferred automatically and simultaneously to remote AutoCAD and renew the same entity of the drawing on each computers.

Figure 11 - Figure 14 are screen captures showing the process two of the designers, Li and WW, conducting a collaborative design using the system developed.

The figures show the CAD drawings, videos, charting board and the list of designers involved (designers $\mathrm{Li}$ and WW in this example). Designer $\mathrm{Li}$, whose video picture is shown on the right, produced the initial CAD drawing and called designer WW's attention, as shown in Figure 11; designer WW confirmed that he received the drawing, as shown in Figure 12, then he modified the drawing as follows: the length of shaft end was changed fro $62 \mathrm{~mm}$ to $45 \mathrm{~mm}$ and the keyway dimension $30 \mathrm{~mm}$ was added, as shown in Figure 13; at the end, designer $\mathrm{Li}$ agreed designer WW's modification, as shown in Figure 14. Please note that those figuers simultaneously appear on the screens of both the designers' computers. 


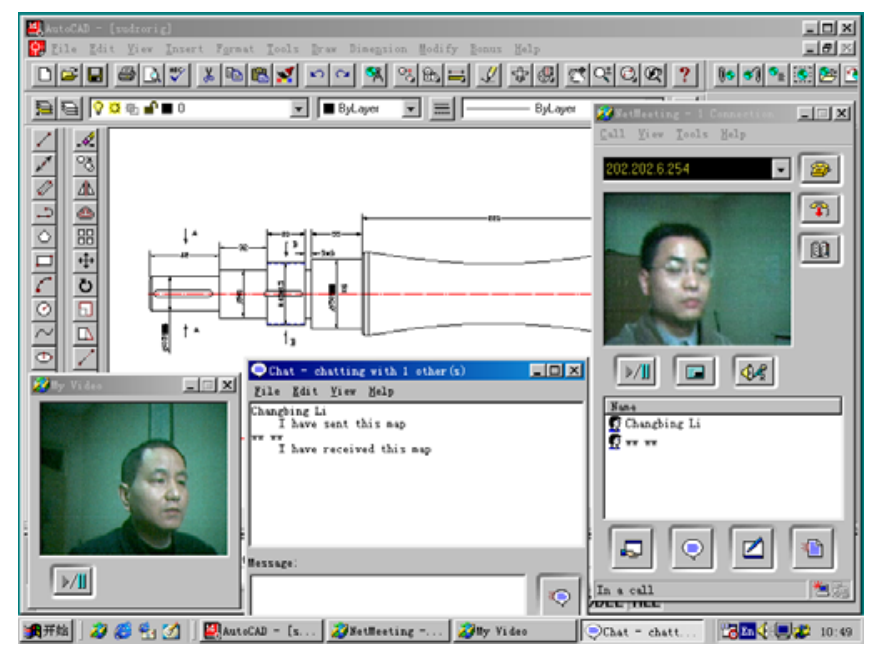

Figure 11. Designer Li (right) produced the initial CAD drawing and called the attention of Designer WW (left)

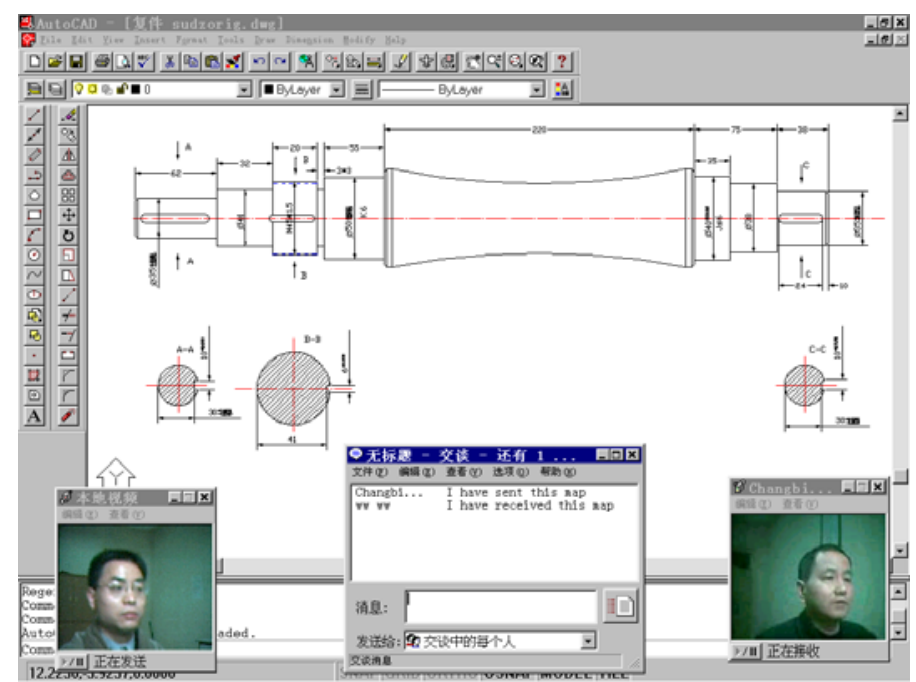

Figure 12. Designer WW (right) confirmed his receipt of the CAD drawing from Designer Li (left).

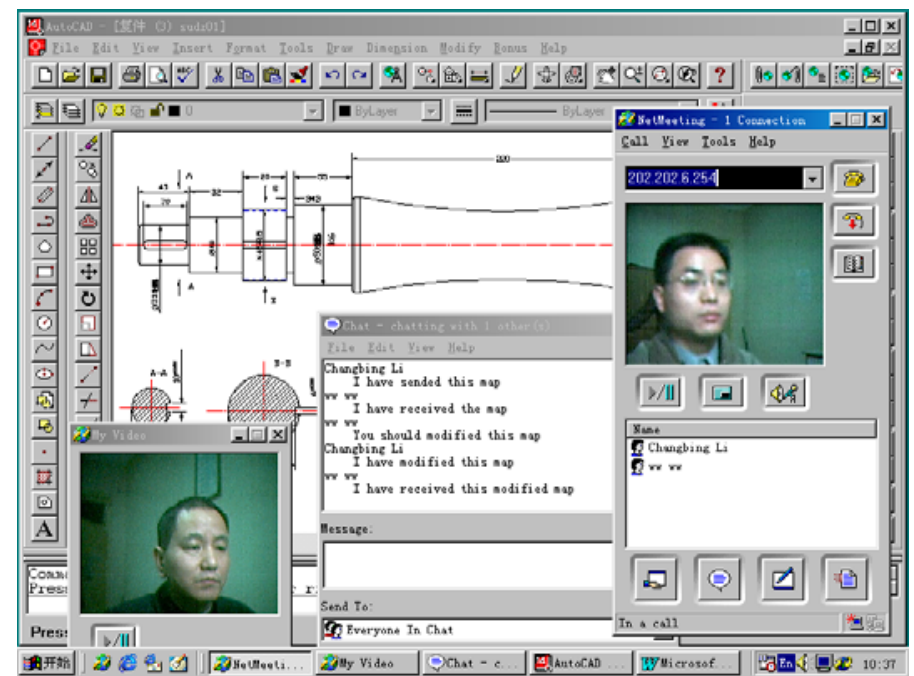




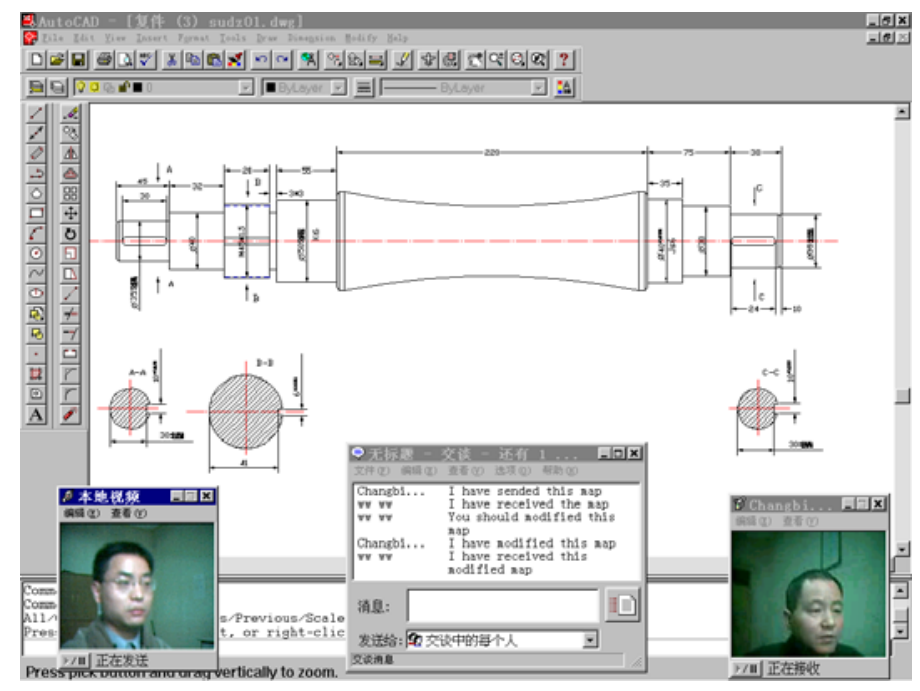

Figure 14 Designer Li agreed the modification

\section{Concluding Remarks}

In the above sections, the framework of network support for the integrated design is presented. The integrated design system integrates various stages within the total design process including product design specification, conceptual design, detail design and manufacture. This is achieved with the aid of integration of various software techniques/packages into a single environment. With in the framework, the main body of the integrated design system and the chief designer, who controls the design process, are located in the main design site. The design tools are accessible for the design team members over the Internet. During the design process, the main design site interacts through the Internet with the client, manufacturer, suppliers and other designers at different locations. With the network support, the integrated design system is utilized on the Internet, so that geographically dispersed designers can collaborate a design task through out the total design process and quickly respond to clients' requests, which enhances the design argilty and speeds up the design process.

The major techniques currently concerned for the network support framework include formulation of the product design specifications via the Internet, integration of online electronic catalogues, remote execution of a large size program and online CAD collaboration. The latter two techniques are described in details.

The remote execution of a large size program technique enables a single copy of a software package to be executed from multiple remote sites, while the online CAD collaboration technique enables multiple copies of a stand along package, with one copy at each site of the network, to interact with each other instantaneously. The former is sever-clients approach where the multiple clients communicate with the sever only but not between the clients themselves; however, there is one copy of the package only, which is economical and easy to update. The latter technique allows the collaborative designers communicate amongst themselves using the stand along package, but a copy of the package for each site is required.

Although the term Internet is used throughout this paper, the techniques developed for the network support for integrated design are applicable for Intranet and Extranet.

\section{Acknowledgement}

The authors acknowledge the following supports for the research reported in this paper: the financial and technical support from the Nottingham Trent University, UK; grants from the programme and organisations supported by the Chinese Government including Cheung Kong Scholars Programme, Natural Science Foundation of China (project No 59875087), and the Education Ministry's Foundation for University Key Academic Staff. The authors are also grateful for the following researchers' contributions: Ms Shuyan Ji, Miss Nariman Amin and Mr Zhiqiang Li. 


\section{References}

[1] S Purba, 2001, 'Architectures for e-Business Systems: Building the Foundations for Tomorrow’s Success', Auerbach Publications (CRC Press), London, ISBN 0-8493-1161-6

[2] D Su, 2000, 'Internet-based Engineering: Applications and Case Studies', The Nottingham Trent University in association with the Professional Engineering Publishing Ltd, ISBN 1-84233-021-7, Nottingham.

[3] G Huang, J Shi and K L Mak, 1997, Internet-based design for X, Proceedings of the $14^{\text {th }}$ International Conference on Computer Aided Production Engineering, Durham, pp 65-71

[4] S Pugh, 'Total Design - Integrated Methods for Successful Product Engineering', Addison Wiley, London 1990

[5] P Y Pan, K Chen and D K Harrison, 2000, 'An AI and Internet-based agile system for rolling bearing design', in D Su (ed.), 'Internet-based Engineering: Applications and Case Studies', published by the Nottingham Trent University in association with the Professional Engineering Publishing Ltd, ISBN 1-84233-021-7, Nottingham, pp 29-60.

[6] N Amin and D Su, 2001, 'Development of on-line electronic gear catalogue using Java database connectivity’, International Conference on Mechanical Transmission, 5-9 April, Chonqing, China, pp 137-141

[7] British Standard 436, Spur and helical gears: Part 3. Method for calculation of contact and root bending stress limitations for metallic involute gears, British Standard Institution, 1986

[8] D. Su and N. Amin, 2000, “ Gear Design Optimisation over the Internet”, in D Su (ed.) Internet-based Engineering: Application and Case Studies, Professional Engineering Publishing Ltd and The Nottingham Trent University, pp61-80.

[9] Microsoft web of NetMeeting, http://www.mircosoft.com/netmeeting (accessed in September 2000)

[10] J Gausemeier, 1996, Global engineering network - weltweiter Informationsverbund zur Staekung der Innovationkraft in Produktentwicklungsprozessen. VDI-Berichte, 1302, 39-52.

[11] D Su and M Wakelam, 1998, 'Intelligent hybrid system for integration in design and manufacture', Journal of Material Processing Technology, Vol 76 (1998), Elsevier Science S A, pp 23-28

[12] D Su, 1999, 'Design automation with the aids of multiple artificial intelligent techniques', Concurrent Engineering - Application and Research Journal, Vol. 7, No1, March 1999, Technomic Publishing Co. Inc., pp 23-30

[13] D Su and M Wakelam, 1997, 'Intelligent integrated system for the design of power transmission systems', proceedings, International Conference on Mechanical Transmissions and Mechanisms, 1 - 4 July, Tianjin, China, pp 1010-1014

[14] D Su and D I Goliski, 1999, 'A computer aided design and learning package and its implementation on the Internet', Journal of Software for Engineering Education, Issue 16, CTI Engineering, pp11-15.

[15] Q Peng and R Hall, 2000, 'Integrated design and manufacture for SME s through a remote web-centered interface', proceedings of the International Conference on Gearing, Transmissions and Mechanical Systems, 3-6 July, Nottingham, pp 376-386.

[16] H Mauer, 2001, 'New aspects of eLearning', http://www.icsc-naiso.org/conferences/nl2002/keynotes.html, (accessed in September 2001)

[17] D Su, and N Amin, 2001, 'A CGI-based approach for remotely excusing a large program for integration of design and manufacturing over the Internet', International Journal of Computer Integrated Manufacturing, Vol 14, No.1, pp 55-65.

[18] D Su, S Ji, N Amin and J B Hull, 2002, ‘Multi-user inveronment for gear design optimisation’, International Journal of Computer Integrated Manufacturing (same issue as this paper)

[19] A Stevens, “Kicking and Scripting: JavaScript and CGI”, Dr. Dobb’s Journal, April 1997, pp. 92-114. 